Revista Posgrado y Sociedad

Sistema de Estudios de Posgrado

Universidad Estatal a Distancia

ISSN 2215-2172

Costa Rica

revistaposgradoysoci@uned.ac.cr

\title{
Las Competencias Gerenciales en la Gestión de Instituciones de Educación Superior
}

\author{
Management skills in managing higher education institutions \\ Minerva Aristimuño V. \\ Wilfredo Guaita \\ Universidad Nacional Experimental de Guayana \\ Ciudad Guayana, Venezuela \\ Carlos Rodríguez M. \\ Universidad Politécnica de Madrid. \\ Madrid, España
}

Volumen 11, Número 2

Setiembre 2011

p. 66-81

Recibido: Marzo, 2010

Aprobado: Marzo, 2011 


\title{
Resumen
}

Esta investigación tiene como objetivo estudiar los componentes de las competencias gerenciales requeridas por directivos o gestores de las Instituciones de Educación Superior (IES). En este sentido, se determinan, de acuerdo a la percepción de los docentes que han desempeñado cargos de dirección en las IES estudiadas, cuáles deberían ser las competencias de los cuadros directivos o gerenciales y como se interrelacionan estas competencias entre sí. Dado que el objeto de estudio son los docentes que tienen o han tenido cargos de autoridad en las IES estudiadas en sus respectivas organizaciones para-universitarias y/o gremiales, se determinó que la población total está constituida por 69 docentes, por lo cual no se calculó una muestra probabilística puesto que es un número pequeño de individuos. El instrumento diseñado fue sometido a la validación de expertos. Se calculó su confiabilidad obteniéndose un valor del alpha de Cronbach de 0,76, considerado como aceptable. El procesamiento de los datos permitió obtener las competencias gerenciales requeridas por los cuadros directivos, asi como las competencias de carácter personal y organizacional. Los resultados encontrados se convierten en insumos para el diseño de programas de formación, desarrollo y gestión de los directivos universitarios.

Palabras clave: Competencias Gerenciales; Formación y Desarrollo; Gestión Universitaria

\begin{abstract}
This research aims to study the components of the managerial competencies required by managers of Higher Education Institutions (HEIs). In this sense, according to the perception of teachers who have held leadership positions in the higher education institutions studied, the responsibilities of senior positions or management and how these skills interrelate with each other are determined. Since the object of study are instructors who have or have had positions of authority in the higher educational institutions studied in their respective organizations, universities and/or related associations, it was determined that the total population consists of 69 teachers. Given the small number of individuals a probability sample does not need to be calculated. The questionnaire designed was subjected to expert validation. Reliability was calculated yielding a Cronbach's alpha value of 0.76 which is considered acceptable. The data processing yielded the managerial competencies required by senior managers, as well as the personal and organizational competencies. The results obtained become inputs for the design of training, development and management programs of university leaders.
\end{abstract}

Keywords: Management Competencies, Training and Development, University Management. 


\section{Introducción}

En las últimas décadas, las universidades se han visto en la necesidad de adecuar sus procesos, para adaptarse a los nuevos eventos del mundo globalizado. Ello debe promover su transformación en organizaciones que innovan y aprenden de sus propias experiencias.

Las universidades están cambiando en función de las exigencias de la sociedad. $\mathrm{Su}$ rol pasivo y de respuesta a largo plazo ya no es pertinente con las nuevas realidades y presiones del entorno, Monagas(2006), Palomares, García, Castro(2008), Armengol, Castro(20032004), Reddy (2008), consideran que las universidades deben jugar un nuevo papel, las presiones y desafíos políticos y económicos, tecnologías de la información, y comunicación entre otros aspectos, originan crecientes demandas, de masificación e internacionalización de la investigación y educación, así como de una mayor calidad, cuyos resultados deben ser cuantificables y generadores de beneficios económicos a la sociedad

En este sentido, de acuerdo a Gutiérrez (2007), la gestión universitaria se estudia desde diferentes aproximaciones. Por un lado se plantea como un modo específico de gestión organizacional y otras veces es vista como un elemento muy particular de la realidad universitaria o mundo universitario.

En líneas generales, plantea el autor, que en función de las posturas paradigmáticas que sean abordadas, con respecto al concepto del término "gestión" puede ser visto como: proceso administrativo, de gobierno, dirección, gerencia o la combinación de ambos términos.

Desde el punto de vista práctico, la palabra gestión es utilizada para el establecimiento de los objetivos de una institución, las estrategias y los medios para lograrlos, y los procesos para llevar adelante las actividades que permitan alcanzar los objetivos con la aplicación de los medios o instrumentos.

La administración es un elemento fundamental de los procesos de gestión, es improbable llevar adelante aplicación de instrumentos para lograr objetivos, sin los mecanismos económicos que los conforman. En este sentido la administración es un factor preponderante dentro de la gestión.

La forma como se hace gestión en las instituciones de Educación Superior, incide en su éxito o el fracaso. La Gestión o dirección de estas instituciones está determinada por las competencias o elementos gerenciales que manejan sus cuadros directivos, quienes en la gran mayoría de las universidades del mundo son sus académicos, elegidos por elección popular y por compromisos políticos.

Estos directivos, aun teniendo profundos conocimientos en su área profesional y una vasta experiencia docente e investigativa, pueden carecer de competencias, experticia y conocimientos gerenciales, lo que podría dificultar el logro del engranaje de los distintos subsistemas universitarios para dar respuestas a las exigencias del entorno. La competitividad en cuanto a demanda de servicios educativos de calidad, 
actualización y adecuación de estructuras funcionales y tecnológicas, está obligando a los cuadros de dirección a desarrollar diversas estrategias para poder diferenciarse de sus competidores.

Por lo tanto, se requiere que estos cuadros directivos flexibilicen sus formas de actuar, acorde con las nuevas realidades; ello lleva implícito un trabajo interdisciplinario, con personas competentes, con dominio de los procesos y como elemento clave con gran capacidad crítica y reflexiva para aprender de los errores y sobre todo para gestionarse a sí mismo como personas. Este cambio de actuación debe permitirles reflejar y transmitir, conductas centradas en valores básicos del ser humano, como el respeto, responsabilidad, la honradez, el compromiso entre otros.

Lo anteriormente planteado, conlleva a que quienes gerencian o gestionan las organizaciones universitarias tengan que asumir de manera dinámica, conductas, comportamientos o estilos acordes a las nuevas exigencias del entorno interno y externo de la universidad y para ello requieren del desarrollo de competencias.

Rodríguez (2006), cita a Spencer y Spencer, quienes plantean "Las Competencias están compuestas de características que incluyen: motivaciones, rasgos psicofísicos y formas de comportamiento, autoconcepto, conocimientos, destrezas manuales y destrezas mentales $\mathrm{o}$ cognitivas" (p. 3).

En este mismo orden de ideas, el autor cita a Boyatzis, quien considera que una competencia puede ser "una motivación, un rasgo, una destreza, la autoimagen, la percepción de su rol social, o un conjunto de conocimientos que se utilizan para el trabajo" (p. 3). Las competencias se fundamentan en las motivaciones de las personas, están asociadas a algunos rasgos que se manifiestan en el carácter, la concepción que tengamos de nosotros mismos, nuestras actitudes o valores, incluso los conocimientos, capacidades cognitivas o conducta manifiestas. Por ello se dice que son características que pueden ser evaluadas, y marcan la diferencia en el desempeño de un trabajador a otro. Además, ocasionan que su trabajo lo realicen de forma exitosa sobrepasando las exigencias, cuando se compara con otro empleado que solo se adecua a los requerimientos mínimos exigidos por el cargo.

Desde el punto de vista conceptual, existen diversos enfoques de lo que son las competencias y algunos autores establecen clasificaciones que las dividen en: genéricas y técnicas, indicando que las primeras se refieren a aquellas que se originan en caracteres o cualidades propios o personales que conciernen a la parte socioemocional cognitiva de las personas.

Por otro lado, al hablar de competencias técnicas, estas se vinculan con los conocimientos técnicos, habilidades y destrezas del área de experticia o procesos que maneja el trabajador. Se infiere así que un académico, para ser exitoso en el desempeño de su gestión universitaria, además de tener un alto nivel de competencias técnicas en su área de conocimiento $y$ en las funciones primigenias (docencia, investigación y 
extensión), debe manejar y desarrollar un conjunto de competencias genéricas, referidas a motivación al logro, toma de decisiones e iniciativa, manejo de efectivas relaciones interpersonales entre otras.

En este mismo orden de ideas, HayGroup (citado por Aristimuño, 2004) reconocida empresa consultora de organizaciones en el área de recursos humanos, identifica de acuerdo a su experiencia una lista de veinte (20) competencias que deben tener los empleados en las organizaciones de acuerdo al cargo que ocupen y las agrupa en función de:

Competencias de logro y acción

Competencias de ayuda y servicio

Competencias de Influencia

Competencias cognitivas

Competencias de eficacia personal

Competencias gerenciales

Esa misma organización afirma que dentro de las competencias gerenciales se encuentran:

Desarrollo de personas: capacidad para emprender acciones eficaces para mejorar el talento y las capacidades de los demás.

Dirección de personas: capacidad de comunicar a los demás lo que es necesario hacer, y lograr que cumplan los deseos de uno, teniendo en mente el bien de la organización a largo plazo.

$>$ Trabajo en equipo y cooperación: capacidad de trabajar y hacer que los demás trabajen, colaborando unos con otros.

$>$ Liderazgo: capacidad de desempeñar el rol de líder de un grupo o equipo. $>\quad$ Gestión del cambio y desarrollo de la organización: Habilidad para manejar el cambio para asegurar la competitividad y efectividad a un largo plazo. Plantear abiertamente los conflictos, manejarlos efectivamente en búsqueda de soluciones, para optimizar la calidad de las decisiones y la efectividad de la organización.

La complejidad de competencias planteadas por Hay Group, crea tensiones adicionales cuando nos referimos al rol de los académicos como gerentes de las organizaciones universitarias; que es el propósito de la presente investigación. Su objetivo principal es analizar los componentes y el sistema de relaciones que debe establecerse en los diferentes niveles organizacionales, para la generación de competencias gerenciales.

Estudios preliminares sobre procesos formativos de los académicos en las universidades, evidencian debilidades y carencias en los programas de formación y desarrollo de contenidos dirigidos a desarrollar competencias en el área gerencial, como ejemplos los estudiados por Aristimuño (2007); Sánchez (2001); Duarte (2004); Romero (2004); Argudelo (2002); Pasek, (2002) y González (2004).

Duarte (2004); parte de la propuesta de un programa para la formación y perfeccionamiento del profesor como docente partiendo del marco institucional que incluye la misión, objetivos y filosofía educativa de la universidad, llegando a determinar el perfil de formación que engloba las competencias y los aprendizajes asociados a esas competencias, para que puedan ejercer en forma idónea la 
función docente. Estas premisas se orientan a un enfoque de integralidad, holístico y dialógico de la función del académico.

Con respecto a los requerimientos que debe tener un gerente, Romero(2004); presenta un análisis de los cambios que son necesarios para transformar la administración pública venezolana y el tipo de gerente o posturas gerenciales que deben ser asumidos, resaltando el compromiso social, expone un análisis epistemológico para la formación gerencial y de la categoría gerencia como tal, considerando, que la situación actual que vive Venezuela requiere que estas "bases epistemológicas se fundamenten en una estrategia de producción de conocimientos que sirva de base para una práctica de profunda transformación". (p. 96)

Pasek, (2002), diseña un modelo teórico que denomina interrelacionado, el cual determina y permite comprender y explicar la formación de investigadores; al asumir la formación de investigadores considera dos dimensiones: $L a$ Holológica y la Holopráxica (p. 161). La primera contempla aspectos lógicos y cuantitativos y la holopráxica aspectos cualitativos, incluye lo subjetivo, considerando las vivencias transpersonales dándose un proceso de complementación entre ambas.

Partiendo de una perspectiva socio histórica de lo que es desarrollo humano, González (2004); vincula los principios, diseño y estrategias curriculares de orientación profesional con el desarrollo de competencias profesionales en los estudiantes universitarios, de esta manera el docente debe poseer cualidades específicas muy particulares para poder transmitir y modelar esas competencias a los estudiantes.

La teoría de la complejidad de Morín (2005), posibilita la necesaria comprensión de los elementos objeto de estudio, pues el entender al individuo complejo en función de sus procesos de aprendizaje significa abordarlo integralmente, como un ser bio-psico-social con múltiples interrelaciones inabarcables.

Estos aspectos son identificados plenamente por Morín (2001), quien al señalar lo complejo del hombre afirma que éste es un ser evidentemente biológico...y "es, al mismo tiempo, un ser evidentemente cultural, metabiológico y que vive en un universo de lenguaje, de ideas y de conciencia.

Pero a esas dos realidades, la realidad biológica y la realidad cultural, el paradigma de simplificación nos obliga ya sea a desunirlas, ya sea a reducir la más compleja a la menos compleja. Vamos entonces a estudiar al hombre biológico en el departamento de biología, como ser anatómico, fisiológico, etc., y vamos a estudiar al hombre cultural en los departamentos de ciencias humanas y estudiar al espíritu como función o realidad psicológica. Olvidamos que uno no existe sin el otro; más aún, que uno es, al mismo tiempo, el otro, si bien son tratados como términos y conceptos diferentes". (p 89)

De acuerdo a Ortiz, $M$ et al (2008); "La universidad en tanto organización social vigente como institución de las ciencias, la educación 
y la cultura tomada como objeto complejo de la realidad puede ser entendida como tal desde la ciencia de sistemas o sistémica. La mirada sistémica se focaliza en el todo organizado, teniendo en cuenta la naturaleza multidemnsional y multinivélica de los sistemas complejos. Las organizaciones y su Management encuentran en ello enormes beneficios". (p. 2)

En este mismo orden de ideas de acuerdo a Ortiz, $\mathrm{M}$ et al, se han realizado grandes esfuerzos para la construcción de teorías y modelos, que tienen en cuenta las características invariantes de los sistemas complejos. Conciben los autores que "el enfoque sistémico se ha convertido en la base científica para las nuevas ciencias de Management que han generado esfuerzos integrativos y holísticos para permitir el diseño, control y el desarrollo de organizaciones y de sistemas sociales en general," acotando que "las universidades son un caso particular de entidad social. Son organizaciones complejas que necesitan ser gestionadas sistémicamente para elevar su excelencia académica en el contexto local, nacional y mundial."

Thompson (2004); considera que uno de los de los desafíos del estudio de los procesos educativos, en ello se abarca la gestión y gerencia, es superar el estudio del conocimiento disciplinar y abordar la complejidad e interdisciplinaridad como parte de un proceso más amplio de las organizaciones. Esto indica la ruptura de paradigmas en cuanto a hábitos de la práctica diaria y de los procesos cognitivos, cambios en cultura, valores y replantearse de manera estructural los procesos de gestión universitaria.

Los cuadros directivos universitarios, no solo necesitan tener conocimiento de los aspectos sustanciales del sector educativo y del entorno donde se desenvuelven estas instituciones, sino tal como lo plantean, Martínez et al,(s/F), "entender que estas deben ser manejadas como organizaciones que deben actuar más allá de los sistemas tradicionales de gestión, que siguen siendo válidos pero insuficientes y por tanto ineficientes, y deben buscar factores sostenibles en el tiempo que no sean fáciles de comprar o copiar por sus competidores, que les permitan recuperar la diferencia y les aporten ventajas competitivas". En este sentido el principal factor para la competitividad de las organizaciones es su gente. (p.4)

Así mismo, reviste particular importancia determinar que todo proceso formativo que esté destinado a la generación de competencias ha de especificar, desde el mismo momento de su concepción programática, la manera en la cual se van a evaluar.

El aprendizaje no es entonces un problema individual de cada uno, constituye por tanto una conjunción de condiciones subjetivas, sociales, institucionales y pedagógicas, por lo que las competencias son: "... construcciones y reconstrucciones de cada individuo en el seno de una comunidad, o mejor, son las interacciones de una persona con un colectivo, las cuales le hacen competente en esa clase de saber que el grupo domina", (Gallego, 2003, p. 76). La evaluación de estos aprendizajes 
habrá de contemplar todos estos aspectos de manera simultánea.

La noción de competencia y su evaluación están incluidas en cualquier análisis de la adquisición de habilidades y de su aplicación. Wolf, Beaumon y Eraut y Cole (citados en Arguelles y Gonczi, 2001) indican que las evaluaciones que determinan el nivel de competencia poseen una validez demostrable en la medición del desempeño ocupacional, cosa de la que carecen las pruebas académicas $\mathrm{y}$ psicológicas.

Una práctica óptima en materia de evaluación indica que la valoración de la competencia debe: combinar el trabajo, el aprendizaje y la evaluación; hacer el uso máximo de actividades de desempeño que produzcan evidencias válidas con respecto a una serie de elementos; hacer el uso máximo de evidencias que ocurran de manera natural, a las que se tenga rápido acceso en el entorno de trabajo o de aprendizaje; cuidar porque la presentación de las evidencias sea clara y porque haya referencias cruzadas y cerciorarse de que los sujetos tengan un papel activo en la planificación y en la recolección de evidencias.

Para la operativización de la evaluación basada en competencias, es necesario proceder por criterios y no por normatividad, la autoevaluación desempeña un papel fundamental e imprescindible. Se trata de recolectar información para emitir un juicio sobre el proceso de construcción y reconstrucción de competencias, por parte de los participantes $\mathrm{y}$, con él, poder afirmar si el proceso de formación está produciendo lo que se persigue.

Lo importante a destacar es que para evaluar las competencias se debe tener conceptualmente establecidos los criterios y los indicadores que permitirán alcanzar el logro estipulado.

Un Criterio o Rúbrica es concebido como un recurso que permite evidenciar avances en el proceso de aprendizaje del participante, es decir, convalidar lo que sabe, qué hace y cuáles son sus actitudes. Se denominan criterios de evaluación a aquellos aspectos de los procesos de enseñanza y aprendizaje que se eligen, con fundamentos claros $y$ contundentes, para evaluar.

Para la evaluación de cada competencia es conveniente establecer un máximo de tres indicadores $\mathrm{y}$, "también han de ser tres los aspectos a evaluar para cada indicador" (Gallego, 2003, p. 94). Esta es una recomendación, que sugiere este autor, infiriéndose que tal sugerencia es con el objetivo de facilitar el análisis y evaluación de las competencias.

\section{Aspectos Epistemológicos y Metodológicos}

La investigación se realizo, en Ciudad Guayana, Municipio Caroní, estado Bolívar, Venezuela, en dos universidades públicas (Universidad Nacional Experimental de Guayana y Universidad de Oriente - Unidad Experimental Puerto Ordaz) y dos universidades privadas (Universidad Católica Andrés Bello y Universidad Gran Mariscal de Ayacucho). 
Para generar conocimiento científico en realidades sociales esencialmente complejas, existe la necesidad de contar con un enfoque integral; esto lo específica la investigadora social Rakowsky (1995) en el capítulo final de CONTRAPUNTO, en el cual expresa que un enfoque multidisciplinario puede disminuir el riesgo de elaborar conclusiones erróneas que provienen de investigaciones muy simples.

Así mismo, afirma que si se integran las descripciones e interpretaciones que provienen de diferentes aproximaciones ideológicas, conceptuales y metodológicas, se puede alcanzar la visión texturizada y rica de la compleja y multidimensional realidad: "...la investigación debe incorporar diferentes niveles de análisis para problemas de investigación concretos. Esos incluyen el nivel macroeconómico, la regulación de políticas ambientales, relaciones de poder, comunidades, unidades económicas y hogares de trabajo" (Op. Cit., p. 276).

Moser (1995, p. 6), explica que los estudios realizados en este tipo de organizaciones sociales todavía no se han decidido por una metodología específica, y por ello, cualquier investigador que intente abordar una realidad social debe hacerse la siguiente pregunta: “¿Cómo operacionalizar y conducir la investigación en este sector: conjuntos de datos que emergen de encuestas o censos; o por el contrario, estudios de casos e investigación etnográfica?"

Es por esta razón que los investigadores han escogido como enfoque metodológico la utilización de instrumentos y aproximaciones que pueden estar, simultáneamente en dos paradigmas: el positivista y el cualitativo.

Se parte de la premisa de que es necesario un enfoque cualitativo para conocer en detalle una realidad social; y en una fase posterior, se diseñan instrumentos de recolección de información que permitan la realización de un proyecto de investigación no experimental, con una dimensión temporal transversal, ya que se trata de: "analizar cuál es el nivel o estado de una o diversas variables en un momento dado, o bien, cuál es la relación entre un conjunto de variables en un punto en el tiempo" (Hernández et al, 2006).

En la aplicación de esta premisa, se realizo una entrevista a profundidad a cinco docentes que son en la actualidad autoridades universitarias (dos de la Universidad Nacional Experimental de Guayana, y uno (1) de cada una de las casas de estudios superiores restantes: Universidad de Oriente - Unidad Experimental Puerto Ordaz, Universidad Católica Andrés Bello y Universidad Gran Mariscal de Ayacucho).

A partir del análisis hermenéutico realizado a estas entrevistas, se diseñó una encuesta para identificar las competencias necesarias, requeridas por los Gerente o Gestores Universitarios.

El instrumento de recolección tipo encuesta consta de 25 reactivos con cinco anclajes de respuestas cada uno, a través de una escala tipo Likert con amplitud de 5 ( $1=$ totalmente en desacuerdo, $5=$ totalmente de acuerdo). Dado que el objeto de estudio son los docentes que tienen $\mathrm{o}$ han tenido cargos de autoridad en dichas 
universidades públicas y/o privadas, en sus respectivas organizaciones para universitarias $\mathrm{y} / \mathrm{o}$ gremiales, se determinó que la población total está constituida por 69 docentes, por lo cual no se calculó una muestra probabilística puesto que es un número relativamente pequeño de individuos. El instrumento diseñado fue sometido a la validación de expertos.

Para analizar la confiabilidad se utilizó el Alfa de Cronbach el cual consiste en administrar el cuestionario una sola vez $\mathrm{y}$ produce valores que oscilan entre o y 1 basado en las correlaciones promedio de los ítems, Hernández et al (2006). Su fórmula de es la siguiente (1):

$$
\alpha=\frac{N \operatorname{Pr}}{1+\operatorname{Pr}(N-1)}
$$

(1)

Donde:

$\mathrm{N}$ : es el número de ítems de la escala

Pr: promedio de las correlaciones entre los ítems.

Con la prueba de confiabilidad mediante el Coeficiente de Alfa de Cronbach el valor obtenido fue de 0.76 que fue considerado aceptable.

\section{Resultados}

Las competencias gerenciales que deben tener los académicos se eligieron mediante una integración ecléctica de dos modelos aplicados en ambientes empresariales: el de Hay Group (1996, p. 80) y uno aplicado en la empresa básica CVG FERROMINERA ORINOCO (2006), que se muestra en la tabla (1).
Tabla 1. Competencias gerenciales

\section{Competencias}

\section{Gerenciales}

1.-Autoconfianza

2.-Sensibilidad

interpersonal

3.-Control de cambio

emocional

4.-Empatía

5.-Desarrollo de personas

6.-Trabajo en Equipo

7.-Liderazgo

8.-Gestión del Cambio y

Desarrollo organizacional

9.-Pensamiento Estratégico

10.-Identificación con la

Organización

Resulta conveniente destacar que las competencias gerenciales que se presentaron, para su validación, ante los académicos objeto de estudio, se agruparon de acuerdo a su naturaleza, es decir, son de carácter personal (autoconfianza, sensibilidad interpersonal, control de cambio emocional, empatía);organizacional (desarrollo de personas, desarrollo y cambio organizacional, identificación con la organización) y gerencial (trabajo en equipo, liderazgo, pensamiento estratégico, desarrollo de personal). 
Cuando se hace referencia a las competencias de carácter personal, se trata de agrupar a las competencias vinculadas con sus características personales, que influyen o impactan al resto del grupo y por ende su éxito en el cargo.

Las competencias organizacionales, se pueden definir como la comprensión y adecuación de las personas, a los aspectos de relaciones que impactan a sus compañeros de trabajo, subordinados, sentido de pertenencia con la organización.

Las competencias gerenciales se vinculan a los comportamientos y acciones de los directivos para facilitar la operatividad del grupo del que es parte. Su práctica es lograr que las personas trabajen juntos eficientemente y logren sus objetivos y expectativas en la organización.

Se refiere como resultado general que, en opinión de los docentes encuestados, estas son las competencias que debe tener el directivo universitario, ver Tabla 2.

Así mismo, es importante destacar que en cada bloque de las competencias a desarrollar se le solicitaba a los docentes que, si les parecía, añadieran cualquier número de competencias adicionales que consideraran relevantes. Dentro de los resultados podemos citar a "la responsabilidad" como una de las competencias personales sugeridas.

Los resultados obtenidos en esta investigación, se pueden convertir en directrices para la revisión de los procesos de gestión de las instituciones de educación superior. En este sentido se recomienda diseñar e implementar programas de formación y desarrollo gerencial, en los cuales, dentro de sus contenidos sean consideradas las competencias requeridas por los cuadros directivos identificadas en este estudio.

\section{Conclusiones}

La gestión de instituciones de educación superior, enfrenta grandes desafíos motivados a un entorno altamente complejo y de difícil predicción, que en el futuro aún estará más marcado por los cambios, por sus causas y sus consecuencias. La adopción del enfoque de competencias exige nuevas formas de gestión en las instituciones, que conllevan a desarrollar mecanismos de administración abiertos, que incorporen la cultura del cambio y el mejoramiento continuo.

Con respecto al abordaje de este tipo de estudio desde el pensamiento complejo, es necesario destacar que la reforma del pensamiento y de los procesos en las instituciones de educación superior, deben estar siempre abiertos a la discusión, reflexión acerca de los valores personales y organizacionales en búsqueda de buscar nuevas características del conocimiento complejo de nuestras problemáticas y buscar las estrategias para su solución.

El diseño y puesta en práctica de programas de desarrollo de competencias gerenciales, en los cuadros directivos universitarios, es una necesidad latente en las instituciones de educación superior, a objeto de garantizar la calidad de los servicios prestados por estas instituciones. 
Tabla 2. Competencias del gerente o gestor universitario.

\begin{tabular}{|llll|} 
Competencias & Competencias & Competencias Gerenciales \\
Personales & Organizacionales & \\
\hline Autoconfianza & Compromiso con & la & Liderazgo \\
& Organización & & \\
Sensibilidad interpersonal & Conocimiento Organizacional & Habilidades de dirección \\
Control de cambio & Gestión del Cambio y & Pensamiento Estratégico \\
emocional & Desarrollo organizacional & \\
Empatía & Capacidad de Negociación & Gestión del Talento Humano \\
Trabajo en Equipo & Administración de Recursos & Resolución de Problemas \\
\hline
\end{tabular}

\section{Referencias}

Aristimuño,M (2004) Metodología para determinar Necesidades de Capacitación y Desarrollo del Personal docente adscrito al Departamento de Organización y Gerencia de la UNEG. Trabajo Especial de grado para optar al Titulo de Magíster en Gerencia de Recursos Humanos.

Aristimuño, M (2007) Análisis Dialéctico de Procesos Formativos Universitarios para la Función Gerencial. Caso de estudio: Universidades que hacen vida en el Municipio Autónomo Caroní. Trabajo Especial para acreditar la Suficiencia Investigadora en el área de conocimiento Organización de Empresas, del Departamento de Ingeniería de Organización, Administración de Empresas y Estadísticas de la E.T.S. Ingenieros Industriales. UPM

Argûelles, A. y Gonczi, A. (2001) Educación y Capacitación Basadas en Normas de Competencia: Una Perspectiva Internacional. México: Editorial Limusa, S.A. de C.V.

Argudelo, S. (2002) Alianza entre formación y competencia. CINTERFOR, Montevideo. . Recuperado el 18 de noviembre de 2009 en: http://www.cinterfor.org.uy/public .

Armengol C,Castro D (2003-2004) Análisis de los nuevos escenarios universitarios: Reflexión Previa a los Process de Cambio. Contextos Educativo, 6-7,(2003-2004),137-150. Universidad de Barcelona

Boyatzis R. (1982) The competent manager. New York Wiley \& Sons.

CVG FERROMINERA ORINOCO (2006) Competencias Genéricas Personal Nivel Medio Ferrominera Orinoco C.A. Material mimeografiado.

Duarte, E (2004) Formación y perfeccionamiento del profesor como docente en la Universidad Panamericana. Campus Guadalajara. Recuperado el 20 de noviembre de 2009 en: http://www.unav.es/fyl/tesisjaeng.htm

Gallego B., R. (2003) Competencias Cognoscitivas. Colombia: Cooperativa Editorial Magisterio.

González M.(2004) La formación de la competencia profesional en la Universidad. Reflexiones y experiencias desde una perspectiva educativa. En Universidad 2004. 4ta Convención Internacional de Educación Superior. La Habana, 1ro al 5 de febrero de 2004 [CD-ROM]

Gutiérrez, Ricardo Raúl (2007) ¿Pueden las universidades estar sujetas al principio de eficiencia en su gestión? Departamento de Economía. Universidad Nacional del Sur. 


\section{Aristimuño, Guaita \& Rodríguez}

Haygroup (1996) Las Competencias: Clave para una Gestión Integrada de los Recursos Humanos. Ediciones Deusto S.A.

Hernández, R., Fernández, C, y Baptista, L. (2006) Metodología de la Investigación. México: McGraww-Hill Interamericana.

Martínez,F, Peñalver,A y Salamanca,J. (S/F) GESTIÓN ESTRATÉGICA DEL CONOCIMIENTO. Recuperado el 20 de noviembre de 2009 en:www.auip.org/archivos/gestionconocimiento.pdf

Monagas, A.(2006) La Gerencia Universitaria ante el Cambio institucional. Revista Visión Gerencial.ISSN 1317-8222. año 004. No 2. Vol 4. .pp151-171.Editor Saber ULA.

Morin, E. (2001) Introducción al pensamiento complejo. (M. Pakman, trad.) Barcelona: Editorial Gedisa, S.A. 8va reimpresión. Trabajo original publicado en 1990.

Morin, E. (2005) Introducción al pensamiento complejo. (M. Pakman, trad.) Barcelona: Editorial Gedisa, S.A. 8va reimpresión. Trabajo original publicado en 1990.

Moser, Caroline (1995) Planificación de Género y Desarrollo. Teoría, Práctica y Capacitación. Lima: Ediciones Flora Tristán - Entre Mujeres.

Ortiz, Maria, Campos, Maria y otros (2008) Organización "universidad” como objeto de estudio transdisciplinario complejidad bajo las perspectivas sistémica y cibernética. Fundación Argentina para el Talento y el Ingenio. Consultora. Sistémica \& Metódica Instituto Galileo Galilei Pakman, Trad. España: Editorial Gedisa S. A. (1a reimpresión). Trabajo original publicado en 2002.

Palomares D, García A, Castro E. ( 2008) Evaluación de las instituciones de educación superior: revisión bibliográfica de sistema de indicadores assessment of higher education institutions: a bibliographic review of indicators' systems. Revista española de documentación científica31, 2, abril-junio, 205-229, 2008.issn 0210-0614

Pasek, E (2002) Modelo Teórico para comprender/explicar la Formación de Investigadores. UNESER. Volumen 3, Año3, Enero-julio 2002. Ediciones de Decanato de Postgrado.

Rakowsky, C. (1995) “Contrapunto: Policy, Researh and the Role of the State”, en: Contrapunto. EE.UU.: State University of New York Press.

Reddy, Y.(2008) Global Accreditation Systems in Management Education: A Critical Analysis. South Asian Journal of Management, 15(2), 61-80. Retrieved September 16, 2009, from ABI/INFORM Global. (Document ID: 1607356371

Rodríguez Trujillo, Nelson. (2006) Selección efectiva de personal basada en competencias.Escuela de Psicología, Universidad Central de Venezuela Psico Consult. Recuperado el 15 de noviembre de 2009 en:http://www.cinterfor.org.uy/public/spanish/region/ampro/cinterfor/temas/complab.

Romero, J. (2004) El nuevo gerente venezolano. Editorial OPSU. Venezuela

Sánchez, J (s/f) Formación inicial para la docencia universitaria. en: Recuperado el 20 de enero de 2010 en:www.rieoei.org/deloslectores/sanchez.pdf

Sánchez J (2001) La Función Docente del Profesor Universitario. Recuperado el 20 de enero de 2009 en :www.rieoei.org/deloslectores/sanchez.pdf.

Thompson Klein, J (2004) Interdisciplinarity and complexity: An envolving relationship. Emergence: Complexity and organization,6,(special Double Issue. Nos 1-2), 2-10 


\section{Nota acerca de los autores}

\section{Minerva Aristimuño}

Licenciada en Sociología. Magíster en Gerencia de Recursos Humanos. Doctorando en Administración de Empresas,Universidad Politécnica de Madrid (UPM). Docente Categoría Agregado. Departamento de Organización y Gerencia. Universidad Nacional Experimental de Guayana (UNEG). Centro de Investigaciones Gerenciales de Guayana. UNEG. Ciudad Guayana. Venezuela.

Correo electrónico: aminerva@uneg.edu.ve

\section{Wilfredo Guaita}

Ingeniero Industrial. Doctor en Administración de Empresas. Vicerrector Administrativo, Universidad Nacional Experimental de Guayana (UNEG). Docente Categoría Asociado. Departamento de Ciencia y Tecnología. UNEG. Centro de Investigaciones Gerenciales de Guayana. UNEG. Ciudad Guayana. Venezuela.

Correo electrónico: wguaita@uneg.edu.ve.

\section{Carlos Rodríguez Monroy}

Licenciado en Ingeniería Industrial (1973) y Doctorado en Organización Industrial (1991) ambos de la UPM. Profesor de Estrategia Corporativa y de Sistemas de Información para la Gestión en la Universidad Politécnica de Madrid (UPM). Coordinador de programa de Doctorado en colaboración con Universidades de América Latina.

Correo electrónico: cmonroy@etssi.upm.es 